\title{
Mitochondrial nanotargeting in malignancies (Review)
}

\author{
ANAMARIA MAGDALENA TOMŞA ${ }^{1 *}$, ANDREI PICOŞ ${ }^{2 *}$, \\ ALINA MONICA PICOŞ ${ }^{3 *}$ and ANDREEA LIANA RĂCHIŞAN ${ }^{1}$ \\ ${ }^{1}$ Department of Mother and Child, Second Pediatric Clinic, 'Iuliu Haţieganu' University \\ of Medicine and Pharmacy; Departments of ${ }^{2}$ Oral Rehabilitation and ${ }^{3}$ Prosthetics and Dental Materials, \\ 'Iuliu Haţieganu' University of Medicine and Pharmacy, 400012 Cluj-Napoca, Romania
}

Received May 22, 2020; Accepted June 22, 2020

DOI: $10.3892 /$ etm.2020.9023

\begin{abstract}
Malignancies represent a burden for the health system worldwide. Treating them represents a challenge through the prism of the cancer cell behaviour and the serious systemic side effects that usually occur. Both traditional (chemotherapy, radiotherapy and surgery) and associated therapies (immunotherapy and hormone therapy) have reached a plateau. The new trend for the management of malignancies includes nanoparticles (NPs) which are studied for both their diagnostic and therapeutical use. NPs can be designed in various ways, many of them targeting mitochondria causing cellular apoptosis. This review summarizes the main characteristics of NPs that are studied in different cancers to highlight their mechanism of action. Since mitochondria play a key role in the cellular homeostasis, they represent the main target for the experimental current studies. While there are NPs approved by the FDA for clinical use, most of them are still under extended research and still need to prove their efficacy and biocompatibility, preferable with minimal systemic side effects.
\end{abstract}

\section{Contents}

1. Introduction

2. Malignancies in the current era

3. Nanotechnology and malignancies

4. Nanoparticles and mitochondria

5. Conclusions

Correspondence to: Dr Andreea Liana Răchişan, Department of Mother and Child, Second Pediatric Clinic, 'Iuliu Haţieganu' University of Medicine and Pharmacy, 3-5 Crisan Street, 400012 Cluj-Napoca, Romania

E-mail: andreea_rachisan@yahoo.com

${ }^{*}$ Contributed equally

Key words: mitochondria, nanoparticles, cancer, malignancy, theranostic

\section{Introduction}

Malignancies represent a major health issue, with a dramatic rise in prevalence in the last decades. In cancer patients the treatment is usually multi-modal, bringing together surgery, chemotherapy, radiotherapy, immunotherapy and sometimes hormone therapy. All of them present serious systemic side effects, and the results are not satisfactory at all times. If the patient survives, there is always the risk of recurrence and developing a new type of cancer later on. This brings up the need to develop new therapies, aiming for improved efficacy with minimal side effects at a minimal cost of production. The need to establish a personalized medicine as a mean of providing targeted delivery of the therapeutic substances based exactly on the type of cancer that each patient has becomes acute. Nanoparticles (NPs) are currently used in physics, material sciences, chemistry, biology and also medicine. The nano-oncology field has registered an impressive progress since NPs can be used in the diagnosis and treatment of cancer (1-5).

This review aims to clarify the use of NPs in the treatment of malignancies. Even though some NPs are already used in the clinical practice, there is still the need to design new molecules in order to obtain therapeutic effect with insignificant side effects and low systemic toxicity.

\section{Malignancies in the current era}

The overall impact of malignancies. Malignant tumors are characterized by an accelerated proliferation of their abnormal cells which grow beyond their physiological limits and which can also invade other organs. Early detected cancer has the best prognosis, with a 5 -year surviving rate approximately $70 \%$ for colon cancer and higher than $75 \%$ for breast cancer. Even if the prevalence of digestive cancers has increased dramatically in the last years (+8\% every 5 years), so has the surviving rate, which has grown from $34.2 \%$ (1976-1980) to 59.6\% (2001-2005) (6-8).

Even though most malignancies are curable only when they are discovered in their early stages, some of them are curable even when they are found in a disseminated stage: testicular seminoma, some types of leukemia and lymphoma (especially in pediatric patients). Nevertheless, some malignant tumors, such as pancreatic cancer, have little potential of cure, most 
of them being diagnosed in a late stage with a rapid evolution towards death which is difficult to attenuate. For this type of cancer surgical resection remains the only curative treatment, with a success rate of only $18-25 \%(9,10)$.

Since each type of cancer needs a different approach in therapy, a correct diagnosis is mandatory. The major therapeutic methods that are used the most are represented by surgery, chemotherapy and radiotherapy.

Existing treatments and their limits. Applying a single method of treatment in a cancer patient is, usually, ineffective. Chemotherapy presents a high rate of intolerance and failure which is due to the fact that it acts on both cancerous cells and healthy cells, causing serious systemic side effects. On the other hand, radiotherapy is incapable of successfully treating localized tumors or eliminating all the loco-regional recurrences because cancerous cells may present primary resistance to the ionizing radiation. Neither radiotherapy, nor chemotherapy could highly improve the survival rate, and the results which surgery brings per se are poor if they lack the complementary effects of radio-chemotherapy $(11,12)$.

In cancer patients, surgery can be either curative or palliative. Curative surgery aims for a complete removal of the tumor (radical surgery), with microscopic margins negative for malignant cells, while trying to preserve the organ functions (conservative/non-conservative surgery). If a complete removal of the tumor is not possible, surgical excision with microscopic/macroscopic (debulking) margins positive for malignant cells is also an option, followed by local radiation of the remaining tumor or of the tumor bed, offering the patient a real chance of survival. Palliative surgery is used for life-threatening complications caused by the tumor (intestinal occlusion, fistulae, hemorrhage) aiming to save the patient's life or it can be done to improve the quality of life. Surgery also offers the possibility to remove metastases in selected patients, improving survival. When surgical interventions are followed by a change in the physical appearance of the patient, reconstructive surgeries can be done once the cancer issue has been solved (13).

Hormone therapy can be used for treating tumors in which hormones play a key role in growth and development: melanoma, meningioma and laryngeal, vaginal, ovarian, pancreatic, but also in gastrointestinal tumors. Still, those tumors can present primary resistance to the hormone therapy or even secondary resistance to it. The best results have been obtained for tumors in which sexual hormones sustain their growth: breast cancer, endometrial cancer and prostate cancer. The effects of hormone therapy can be seen at the targeted organ level, but the adverse effects can be found in other parts of the body. Even so, patients tolerate hormone therapy better than chemotherapy. Due to the fact that some tumors do not present hormonal receptors, they will not respond to this therapy, thus a vigorous selection of the patients must be made (14).

Immunotherapy aims to destroy cancerous cells with a high specificity by activating the defensive mechanisms of the host so that it can recognize the cancerous cells as non-self. This is made possible by using immune-active agents such as cytokines, cellular or humoral products, vaccines, gene fractions inoculated by transfection with or without immunological influence from other agents or drugs. Still, so far, immunotherapy has not been able to bring major improvements in patient care $(1,15)$.

The effectiveness of the standard cancer therapies (radiotherapy, chemotherapy and surgery) has reached a plateau for most cancers because of their risk of recurrence and development of new types of disease. Therefore, new therapeutic approaches must be explored, aiming for improved efficacy, minimal side effects and a minimal cost of production. The need to establish a personalized medicine as a mean of providing targeted delivery of the therapeutic substances based exactly on the type of cancer that each patient has become acute $(1,11-15)$.

\section{Nanotechnology and malignancies}

NPs: Overview. The knowledge, techniques and approaches in nanotechnology touch disciplines such as physics, material sciences, chemistry, biology and medicine. Up to now, nanotechnology showed its effectiveness in three large medical domains: therapeutics, diagnosis/imaging and regenerative medicine. In recent years, impressive progress was recorded in the nano-oncology field, with the development of NPs which can be used in the diagnosis and treatment of cancer. The newly developed nanosystems may have decisive influence in cancer therapy in the next decade through new formulas for early detection of cancer, highly sensitive and selective imagistic agents, targeted therapies with minimal systemic side effects and development of minimal invasive techniques $(4,5,16,17)$.

Up to now, a large variety of NPs that can circulate through the blood flow and target tumors have been developed. Unlike low molecular weight drugs, NPs are multifunctional due to the fact that their structural parts, shell and core, can be modified in order to simultaneously offer them different properties. This way and by taking advantage of the large surface:volume ratio of the NPs, unique biological properties can be given by combining luminescence, magnetism, plasmonic heating and even transport and delivery of active substances. Functionalized NPs can be built with high precision to make them capable of absorbance, binding or transport with high effectiveness of different types of molecules (drugs, fluorescent molecules or groups, DNA, RNA, proteins, glycans). These NPs can be designed in order to be compatible with different ways of administration $(4,5,16,18)$.

Complex multifunctional NPs are, in fact, nanosystems which have the capacity to provide therapeutic molecules directly to the cancer cells, therefore diminishing their toxicity on the healthy cells and decreasing their systemic side effects. Due to the fact that tumors present a poor lymphatic drainage and a neovascularization system with dilated, irregular and frail blood vessels, nanosystems are able to extravasate easily and agglomerate at the tumor level. The selective accumulation of the NPs in the tumoral microclimate is known as enhanced permeability and retention effect (EPR) and it makes tumor targeting possible, with imagistic and therapeutic purpose. Alternatively, functional molecules such as antibodies, antigens, peptides can be attached to the surface of the NPs. This attachment will play a role as a target group. The form, size 
Table I. Main types of nanoparticles.

\begin{tabular}{|c|c|c|}
\hline Nanoparticles & Type & Advantages \\
\hline First generation & $\begin{array}{l}\text { Liposomes } \\
\text { Polymeric platforms }\end{array}$ & Increase solubility of hydrophobic molecules \\
\hline Second generation & $\begin{array}{l}\text { Central core particle with magnetic/optical properties } \\
\text { Functionalized surface (antigens/antibodies) }\end{array}$ & Localization/manipulation from the outside \\
\hline Third generation & $\begin{array}{l}\text { Nanostructured core } \\
\text { Functionalized surface (pores/polymers/biomolecules) }\end{array}$ & $\begin{array}{l}\text { Gate system control } \\
\text { Tumor targeting } \\
\text { Substance delivery } \\
\text { Tracking from the outside }\end{array}$ \\
\hline
\end{tabular}

and surface properties of NPs can be modified according to necessity, allowing the particle to maintain its stability and transport capacity $(4,5,18-22)$.

NPs and cancer treatment. The first generation of NPs designed against malignant tumors includes soft NPs, such as liposomes and polymeric platforms, which have already been approved for clinical use. Their main advantage is their capacity of increasing the solubility of hydrophobic molecules, improving their delivery time and effectiveness. The second generation of anticancer NPs is represented by soft NPs that have been functionalized with antigens or antibodies that are attached to their surface. Systems based on inorganic NPs are in more wider use due to the fact that they can utilize a central core particle with magnetic properties or optical properties which can improve their localization and manipulation from the outside of the body by simply using a magnetic field or irradiation. The third generation of bioactive NPs combine a nanostructured core with either pore systems for storage or with molecular fragments attached to the surface (polymers, biomolecules) which allow a gate system control as a response to external stimuli. These NPs are capable of tumor targeting, substance delivery and tracking from the outside, therefore marking the beginning of the theranostic domain (Table I) $(4,19,23,24)$.

Theranostic NPs. Theranostic is a relatively new, revolutionary term in the cancer study field. It combines two essential words: 'therapeutic' and 'diagnostic', therefore it refers to those NPs that can be used for both diagnosis and treatment in the same patient. This new domain offers the possibility to identify the responders as well as the non-responders to the treatment during its administration, allowing the adjustment of the treatment for each individual patient. Consequently, the diagnosis, the treatment and the monitoring of the cancer patient can be completed using a single approach. As theranostic agents, metal NPs present with more advantages because of their unique physical and chemical properties: plasmonic resonance, fluorescence enhancement, superparamagnetism, photoluminescence, enhancement of the catalytic activity, as well as producing reactive oxygen species (ROS). Photoluminescence and superparamagnetism are mostly useful in the imagistic field, while their capacity of releasing ROS is useful in extinguishing malignant cells. This outcome may be attained by other means and mechanisms (i.e. hyperthermia and photothermal effect), depending on the type of metal that is used, the size of the NP, its form, as well as its surface properties $(18,21,23,24)$.

Toxicity of NPs. The mechanism of action of the NPs differs with their structure. Their toxicity has to be well acknowledged and understood in order to be able to determine the potential risk they might have on human health. Their main way of action is represented by producing ROS. Still, an excess of oxidative stress (OS) can be harmful as long as the cells can trigger their own protective mechanisms against it. The defensive antioxidant mechanism of the cells is both enzymatic, as well as non-enzymatic and it is composed mainly of glutathione peroxidase, catalase and superoxide dismutase. But when those protective mechanisms are not able to re-establish the redox equilibrium of the cell, the macromolecules (proteins, lipids, DNA) are damaged. The irreversible transformations that might take place may lead to necrosis and/or apoptosis of the cell. The excess of OS can trigger a pro-inflammatory response, with interleukin-2 and tumor necrosis factor- $\alpha$ activation. The chronic inflammation might lead to the development of atherosclerosis, pulmonary diseases or it may even cause other cancers, but this concept is validated only for NPs that produce ROS as their main way of action. Other NPs affect calcium homeostasis by increasing the calcium influx or by inhibiting calcium sequestration, thus affecting the cellular metabolism, signal transduction and gene expression. The systemic toxicity of the metallic NPs can be diminished by targeting a specific site or by using biodegradable polymers as reduction and functionalizing agents. Metallic NPs may also show toxicity directly through their dissociated ions (24-26).

Multiple studies have been conducted to establish the effect of NPs on different types of digestive cancers. For the gastro-esophageal cancer magnetic iron NPs, polypeptidic NPs and triblock copolymers NPs have been studied. Polymeric nanospheres are being designed against colon cancer together with NPs made of semiconductor materials and GNPs against many other types of other cancerous cells. Ferric oxide NPs and ferrous oxide NPs covered in gold present a huge potential as theranostic agents in pancreatic cancers and may be able of changing its prognosis in the next decade (27-36). 


\section{Nanoparticles and mitochondria}

Mitochondria: An overview. Mitochondrion represents membrane-bound organelle found in the cytoplasm of the eukaryotic cells and it is responsible of generating energy in form of adenosine triphosphate (ATP). These organelles store calcium as part of their cell signaling activities. Mitochondria are able to mediate both the growth and death of the cell. However, the dysfunction of the mitochondrion can be caused by genetic mutations of their proteins or by OS, and it may lead the development of new diseases such as neurodegenerative diseases, diabetes and obesity. Unlike healthy cells, the Warburg effect states that malignant cells use glycolysis for energy production, rather than the tricarboxylic acid cycle. This phenomenon was attributed to mitochondrial dysfunction in malignant cells, especially in the mitochondrial respiratory chain, suggesting that this forces cancer cells to use glycolysis (37-39).

It is well known that NPs cause cytotoxicity by inducing the production of ROS. Mitochondria represent the main intracellular source of ROS which are the primary endogenous agents that alter the DNA, lipids and proteins, contributing to the development of different diseases. Peroxisomes are also involved in ROS production and clearance. If their function is impaired, the result is an increased ROS level inside the mitochondria, which causes mitochondrial damage and further aggravates the clearance of ROS. This leads to promotion of the development of malignant tumors. Chromosomal instability, as well as genome instability and mitochondrial genome mutations can be triggered by ROS production, promoting further ROS formation and signaling pathways such as mitogen-activated protein kinase (MAPK) and PI3K, in tumors. The survival and proliferation of tumor cells can be promoted by ROS under hypoxia conditions. Hypoxia-inducible factors (HIFs) are upregulated to further promote oncogene expression. Excessive ROS causes OS and subsequent damage and cell death, while normal levels of ROS found in healhty cells work as signaling molecules that activate physiological intracellular pathways leading to autophagy and apoptosis. It has been reported that two metallic NPs (Co and Ni) led to glutathione depletion and ROS generation, thus confirming that OS has been implied in the response against these particles. Even though not all NPs have electron configurations or surface properties that allow spontaneous generation of ROS, the interaction between them and the cellular components is what triggers the generation of OS. Therefore, the evaluation of ROS production represents a valid test for comparing the relative toxicity of different NPs (39-42).

Mitochondria are not only the main ROS source of the cell, but also the organelles responsible for cell death. The loss of membrane potential of the mitochondrion releases the intramitochondrial components into the cellular cytoplasm which will trigger the apoptotic cascade. It has also been proven that the mitochondrial membrane potential plays an important role in initiation of autophagy. Autophagy represents a cellular degradation process which is lysosome-dependent, serves the dysfunctional organelles and eliminates different substances from the cell. Autophagy represents not only a process that helps the cells to survive, but also a mechanism of inducing cell death. NPs have been previously proposed as a new class of autophagy activators with a response that depends on the size and shape of the particles. It has been stated that autophagy induced by NPs may lead to cellular cytotoxicity. In cancer cells, mitochondria have to be fully functional but even so, germline and somatic mtDNA mutations have been reported in various types of cancers. It is considered that there could be two classes of mtDNA mutations in malignant cells: those that damage OXPHOS and further stimulate neoplastic conversion, and mutations that facilitate malignant cell adaptation to different bioenergetic environments. Also, mutations in mitochondrial enzymes that are encoded in the nuclear DNA may be present in malignant cells, such as SDH, FH, IDH1 and IDH2 mutations (43-45).

NPs targeting mitochondria. The use of NPs in order to trigger specific organelles is usually limited by the endosomal pathway which serves as a protection barrier. This phenomenon makes it difficult to trigger organelles, making it necessary to validate the presence of the NPs outside the lysosomes and inside the targeted organelle $(46,47)$. Guo et al (46) validated the mitochondrial localization of mitochondria-targeting magnetic composite nanoparticles (MMCNs) by different methods. Firstly, the presence of the MMCNs at the mitochondrial site was confirmed using the Mitotracker kit which offers a green fluorescence visible with confocal fluorescence microscopy. The fluorescent light emitted by the mitochondria overlapped the fluorescence produced by MMCNs. Secondly, an immunocytochemical coloration (Cy 7) was used as a load to follow the NPs, confirming their mitochondrial localization by using confocal laser scanning microscopy (CLSM). In the third place, the presence of MMCNs at the mitochondrial site was validated using a technique that measures the quantity of $\mathrm{Fe}$ in different cellular compartments and compares the treated cells with cells that have not been exposed to MMCNs. This technique is made possible by the presence of $\mathrm{Fe}$ in the MMCNs. The Fe load in different organelle was measured using ICP-AES (Inductively coupled plasma atomic emission spectrometer) and the results showed a significant accumulation of MMCNs inside the mitochondria.

Liposomes, as a mean of mitochondrial delivery of therapeutic substances, present a huge potential in mitochondrial targeting, especially thanks to their high biocompatibility and non-toxicity. At the same time, the research in this field is one of the oldest and one with the most therapeutic substances that have been approved by the FDA. The first of them, Doxil ${ }^{\circledR}$, was approved by FDA in 1995. In digestive cancers, an interesting study was found focused on liposomes filled with clodronate (CLD) and their role in decreasing tumorigenesis. A mouse model of colon cancer was used injecting CLD or phosphate-buffered saline (PBS) encapsulated liposomes to target macrophages, after polyps developed. The tumor number was reduced in CLD treated mice, with a reduction in gene expression (EMR1), decreased p38 MAPKm STAT3 and ERK signalling, and also decreased macrophage markers (IL-6, IL-13, IL-10, TGF $\beta$, CCL17). The authors concluded that targeting macrophages during late-stage tumorigenesis can be successful in reducing tumor growth. Liposomes loaded with a chemotherapeutic agent was not used, but instead CLD, a non-amino bisphosphonate was used (48). 
Kim et al (49) synthesized nanocomposites (HA-ZnONcs) using zinc oxide ( $\mathrm{ZnO})$ and hyaluronic acid (HA) which they supplementary functionalized with ginsenoside $\mathrm{Rh} 2$ to form Rh2HAZnO. They exposed three different cancer cell lines (A549-lung cancer, HT29-colon cancer, MCF7-breast cancer) and one healthy cell line (HaCaTs-human keratinocytes) to $\mathrm{Rh} 2, \mathrm{HAZnO}$ and Rh2HAZnO. In A549 cells, Rh2HAZnO showed significant damaged mitochondria, with intracellular debris, indicating that the NPs were able to target the mitochondria, causing apoptosis by injuring its outer cellular membrane. Moreover, cell viability was utmost reduced in MCF-7 cells.

Wang et al (50) developed nanomicelles loaded with paclitaxel and functionalized with a mitochondria-targeting group (triphenyl phosphine, TPP). As in the previous study, they also used A549 cells which were exposed to their nanomaterial, showing that the positively charged nanomicelles could be localized at the mitochondrial level at $24 \mathrm{~h}$ after exposure. The outer membrane of the mitochondria was permeabilized, which led to the release of cytochrome $c$ and to the activation of caspase- 3 and caspase- 9 (apoptotic effect), as well as to an inhibition in the expression of Bcl-2 (antiapoptotic effect). They concluded that nanomicelles were able to target the mitochondria and by this, they were able to activate different signalling pathways leading to the apoptosis of cancerous cells.

Novel NPs are being developed, and currently carbon-dot-supported gold $(\mathrm{Au} / \mathrm{CDs})$ are of great interest in the field of cancer treatment. Gong et al (51) used Au/CDs to further develop MitoCAT-g, an OS amplifier. After MitoCAT-g was endocytosed, ROS-generating cinnamaldehyde was released, generating high amounts of ROS inside the mitochondria. Also, gold formed Au-S bonds with glutathione, depleting this antioxidant, thus further amplifying the OS in mitochondria, causing apoptosis of the cell.

The functional and cytotoxic effects of mitochondrial transit of the nanomaterial in vitro. The cytotoxicity of different NPs varies according to their type, therefore individual testing for each type of NP is needed. Metallic NPs and metal oxide NPs present common mechanisms of action such as generating ROS and reactive nitrogen species (RNS), but also the direct action of dissociated ions. When they enter in the blood stream, NPs adsorb proteins, in a progressive and selective manner. The direct consequence of this phenomenon is the formation of a protein corona, which is usually comprised of a soft part and a hard part. The hard part of the corona binds to the surface of the NP, while the soft corona forms on top of the hard corona. The protein corona may cause the aggregation of NPs, may lead to undesired cellular uptake or may trigger immune responses. It may also present positive effects, such as diminished cytotoxicity. The capacity of binding proteins is dependent on the surface functionalization, but also on the positive or negative charges of the NPs (52-54).

The mitochondrion, as the target of the NPs, may augment their cytotoxicity by increasing the intracellular concentration of ROS. ROS are defined as molecules that contain one or more oxygen atoms that present a higher reactivity than that of the molecular oxygen. The mean lifetime of ROS and RNS species measures between $10^{-9}$ and $10^{-6} \mathrm{sec}$, inversely proportional with their reactivity. The most common ROS and RNS species are superoxide, hydroxy peroxyl, hydroxyl, nitric and nitrogen peroxide radicals. In the physiological state, ROS represent vital regulators in different intracellular processes including cell growth, survival, proliferation, apoptosis, cell migration and inflammation. A pathological increase in ROS concentration leads to oxidative alteration of lipids (peroxidation), proteins (amino acids alteration, fragmentation and alteration of protein) and DNA (the occurrence of mutations and damage to gene transcription). As a defense mechanism against the OS, hem-oxygenase 1, superoxide dismutase, catalase and glutathione peroxidase are produced. At higher concentration of ROS species, pro-inflammatory signalling pathways such as JNK and NF- $\mathrm{KB}$ cascade are activated. Higher ROS concentrations that cause prolonged OS lead to changes in the mitochondrial membrane potential causing cell death (25,55-58).

In another study, zinc peroxide NPs $\left(\mathrm{ZnO}_{2} \mathrm{NPs}\right)$ with a hydrodynamic diameter of $66.1 \mathrm{~nm}$ were developed. The particularity of this study was represented by the fact that the NPs were able to deliver exogenous hydrogen peroxide and simultaneously amplify the production of endogenous ROS due to the presence of metallic ions $\left(\mathrm{Zn}^{+2}\right)$. It was shown that zinc inhibited the mitochondrial electron transport chain, increasing the endogenous mitochondrial production of ROS (superoxide and hydrogen peroxide). In a synergistic manner, $\mathrm{H}_{2} \mathrm{O}_{2}$ was released exogenously, leading to a highly effective anticancer therapy. It was concluded that the dissociation behavior of their NPs was pH-dependent, making them more efficient in causing apoptosis in cancer cells with negligible damage to healthy organs, which can be explained by the fact that malignant cells are more susceptible to additional ROS (59).

There are two major pathways through which the cell produces ATP: glycolysis and via the Krebs cycle. The extracellular acidification rate (ECAR) represents a quantifiable index for the glycolytic activity, while the activity of the mitochondrial respiratory chain can be quantified by measuring the oxygen consumption rate (OCR). The two parameters may be used in order to determine how the NPs perform at the cell level: by the impairment of the mitochondrial function or by inhibiting the enzymatically catalyzed redox reactions (60).

Huo et al (61) synthesized silica NPs (MSN) containing a photosensitizer (Ce6) and $\mathrm{W}_{18} \mathrm{O}_{49}$ NPs (WOPNs) which they functionalized with triphenyl phosphonium (TPP, for mitochondrial targeting) and with nuclear localization sequence (NLS, for nucleus targeting), respectively. They further conjugated MSNs [Mito(T)] with WOPNs [Nuc(T)] via a peptide that can be cleaved by Cathepsin B [Mito(T)-pep-Nuc(T)]. The expression of Cathepsin B is known to be abnormally high in various cancer cells. They observed the effect of applying photodynamic (PDT) followed by photothermal therapy (PTT) using laser irradiation with a wavelength of $633 \mathrm{~nm}$ followed by $1,064 \mathrm{~nm}$. The ATP supply of the malignant cells (HCT-116-human primary colon cancer line) was abolished due to the destruction of both organelles. Cancer cells that were exposed to Mito(T)-pep-Nuc(T) and laser irradiated had intracellular levels of ROS 182-fold higher than the control group (HHL-5-hepatocyte cell line). The vulnerability to PDT of the mitochondria proved to 
be dependent on the size and shape of the mitochondria, with the little globule-like mitochondrion being least prone to photodermal therapy, while branched tubule-like mitochondrion being the most vulnerable. Sequential laser irradiation also indicated that the cell death was related to a mitochondrial apoptotic pathway.

A study that also showed the increased production of ROS in the cells exposed to NPs used MMCNs loaded with ICG (indocyanine green), a photosensitizing agent approved by US Food and Drug Administration (FDA). This agent was able to generate ROS after near infrared laser exposure and it was able to partly convert the luminous energy into thermal energy in order to improve the NPs' performance. The cells that had internalized ICG-loaded MMCNs emitted a green fluorescence when they were laser treated, which suggests that these particles were able to generate ROS inside these cells, compared with a control group that had no fluorescence, therefore no ROS production (46).

It is a well known fact that in a healthy cell the programmed cell death occurs through cytochrome $\mathrm{C}$ releasing from the mitochondria under stress conditions. In cancerous cells this phenomenon does not occur, therefore cellular death appears late. Wang et al (50) studied the mitochondrial pathways of apoptosis, demonstrating that releasing cytochrome c represents an important event in a cancer cell exposed to nanomaterials. In their study, as mentioned above, nanomicelles functionalized with TPP for mitochondrial targeting, causing cytochrome $\mathrm{C}$ release which led to apoptosis of malignant cells.

The mitochondria of the cancer cells are usually more vulnerable to disruptions than those in healthy cells because of structural and functional differences that exist between the two. Therefore, therapies that selectively target the mitochondria of cancer cells have as a main purpose the destruction of the tumor without negatively affecting the healthy surrounding tissues $(37,61)$.

\section{Conclusions}

With the rising incidence of cancers, the high mortality rate in some of them (e.g., pancreatic) and the limits that the conventional therapies present, the need to find new therapies is urgent. NPs might represent one of the solutions, which makes them of great interest when it comes to experimental research, with promising results. While first generation NPs are already used in clinical practice, newer generations need to prove that they are effective and safe to use in humans. Unfortunately, more studies need to be done in order to create the perfectly structured nanoparticle that would target and destroy only the cancerous cell, with no harmful effects on the healthy tissues. Until then, malignancies remain a huge burden for the society, with high morbidity and mortality rates.

\section{Acknowledgements}

Not applicable.

\section{Funding}

No funding was received.

\section{Availability of data and materials}

Not applicable.

\section{Authors' contributions}

AMT, AP, AMP and ALR contributed to the acquisition of the data, and the drafting and critical revision of the manuscript for important intellectual content. All authors read and approved the final manuscript.

\section{Ethics approval and consent to participate}

Not applicable.

\section{Patient consent for publication}

Not applicable.

\section{Competing interests}

The authors declare that they have no competing interests.

\section{References}

1. Kruger S, Ilmer M, Kobold S, Cadilha BL, Endres S, Ormanns S, Schuebbe G, Renz BW, D'Haese JG, Schloesser H, et al: Advances in cancer immunotherapy 2019 - latest trends. J Exp Clin Cancer Res 38: 268, 2019.

2. Singh M, Harris-Birtill DCC, Markar SR, Hanna GB and Elson DS: Application of gold nanoparticles for gastrointestinal cancer theranostics: A systematic review. Nanomedicine (Lond) 11: 2083-2098, 2015.

3. Nayak D, Minz AP, Ashe S, Rauta PR, Kumari M, Chopra P and Nayak B: Synergistic combination of antioxidants, silver nanoparticles and chitosan in a nanoparticle based formulation: Characterization and cytotoxic effect on MCF-7 breast cancer cell lines. J Colloid Interface Sci 470: 142-152, 2016.

4. Hock1 PF, Wolosiuk A, Pérez-Sáez JM, Bordoni AV, Croci DO, Toum-Terrones Y, Soler-Illia GJAA and Rabinovich GA: Glyco-nano-oncology: Novel therapeutic opportunities by combining small and sweet. Pharmacol Res 109: 45-54, 2016.

5. Ancuceanu R, Dinu M, Neaga I, Laszlo FG and Boda D: Development of QSAR machine learning-based models to forecast the effect of substances on malignant melanoma cells. Oncol Lett 17: 4188-4196, 2019.

6. Solomon I, Voiculescu VM, Caruntu C, Lupu M, Popa A Ilie MA, Albulescu R, Caruntu A, Tanase C, Constantin C, et al: Neuroendocrine factors and head and neck squamous cell carcinoma: An affair to remember. Dis Markers 2018: 9787831 , 2018.

7. Jooste V, Remontet L, Colonna M, Belot A, Launoy G, Binder F, Faivre $J$ and Bouvier AM: Trends in the incidence of digestive cancers in France between 1980 and 2005 and projections for the year 2010. Eur J Cancer Prev 20: 375-380, 2011.

8. Boda D, Docea AO, Calina D, Ilie MA, Caruntu C, Zurac S, Neagu M, Constantin C, Branisteanu DE, Voiculescu V, et al: Human papilloma virus: Apprehending the link with carcinogenesis and unveiling new research avenues (Review). Int $\mathbf{J}$ Oncol 52: 637-655, 2018.

9. Pei X, Song F and Wang Z: Emerging incidence trends and application of curative treatments of pancreatic cancer in the USA. Medicine (Baltimore) 98: e17175, 2019.

10. Zeng S, Pöttler M, Lan B, Grützmann R, Pilarsky C and Yang H: Chemoresistance in pancreatic cancer. Int J Mol Sci 20: 4504 , 2019.

11. Pellino A, Riello E, Nappo F, Brignola S, Murgioni S, Djaballah SA, Lonardi S, Zagonel V, Rugge M, Loupakis F, et al: Targeted therapies in metastatic gastric cancer: Current knowledge and future perspectives. World J Gastroenterol 25: 5773-5788, 2019. 
12. Hall WA and Goodman KA: Radiation therapy for pancreatic adenocarcinoma, a treatment option that must be considered in the management of a devastating malignancy. Radiat Oncol 14: $114,2019$.

13. Dekker E, Tanis PJ, Vleugels JLA, Kasi PM and Wallace MB: Colorectal cancer. Lancet 394: 1467-1480, 2019.

14. Blundon MA and Dasgupta S: Metabolic dysregulation controls endocrine therapy-resistant cancer recurrence and metastasis. Endocrinology 160: 1811-1820, 2019.

15. Wang S, Cowley LA and Liu XS: Sex Differences in cancer immunotherapy efficacy, biomarkers, and therapeutic strategy. Molecules 24: 3214, 2019.

16. Li Y, Ayala-Orozco C, Rauta PR and Krishnan S: The application of nanotechnology in enhancing immunotherapy for cancer treatment: Current effects and perspective. Nanoscale 11: 17157-17178, 2019

17. Lin W: Introduction: Nanoparticles in medicine. Chem Rev 115: 10407-10409, 2015

18. Saeed M, Gao J, Shi Y, Lammers T and Yu H: Engineering nanoparticles to reprogram the tumor immune microenvironment for improved cancer immunotherapy. Theranostics 9 : 7981-8000, 2019.

19. van der Meel R, Sulheim E, Shi Y, Kiessling F, Mulder WJM and Lammers T: Smart cancer nanomedicine. Nat Nanotechnol 14: 1007-1017, 2019.

20. Raza F, Zafar H, You X, Khan A, Wu J and Ge L: Cancer nanomedicine: Focus on recent developments and self-assembled peptide nanocarriers. J Mater Chem B Mater Biol Med 7: 7639-7655, 2019.

21. Thakur V and Kutty RV: Recent advances in nanotheranostics for triple negative breast cancer treatment. J Exp Clin Cancer Res 38: 430, 2019.

22. Wang L, Huang J, Chen H, Wu H, Xu Y, Li Y, Yi H, Wang YA, Yang $L$ and Mao $H$ : Exerting enhanced permeability and retention effect driven delivery by ultrafine iron oxide nanoparticles with T1-T2 switchable magnetic resonance imaging contrast. ACS Nano 11: 4582-4592, 2017.

23. Gholami A, Mousavi SM, Hashemi SA, Ghasemi Y, Chiang WH and Parvin N: Current trends in chemical modifications of magnetic nanoparticles for targeted drug delivery in cancer chemotherapy. Drug Metab Rev 52: 205-224, 2020.

24. Sharma H, Mishra PK, Talegaonkar S and Vaidya B: Metal nanoparticles: A theranostic nanotool against cancer. Drug Discov Today 20: 1143-1151, 2015

25. Tomsa AM, Alexa AL, Junie ML, Rachisan AL and Ciumarnean L: Oxidative stress as a potential target in acute kidney injury. PeerJ 7: e8046, 2019.

26. Zuberek $M$ and Grzelak A: Nanoparticles-caused oxidative imbalance. Adv Exp Med Biol 1048: 85-98, 2018.

27. Ghosn M, Kourie HR and Tabchi S: Gastrointestinal cancers in the era of theranostics: Updates and future perspectives. World J Gastroenterol 21: 8473-8477, 2015.

28. Zeng Q and Baker I: Iron/iron oxide nanoparticle and use thereof. Patent WO2012036978A1. Filed September 9, 2011; issued March 22, 2012.

29. Abd-Elgaliel WR and Tung $\mathrm{CH}$ : Protease degradable polypeptides and uses thereof. Patent WO2012075241A3. Filed December 1, 2011; issued June 7, 2012.

30. El-Sayed MEH and Yuksel Durmaz Y: Polymeric nanoparticles for ultrasound imaging and therapy. Patent WO2013055791A1. Filed October 10, 2012; issued April 18, 2013.

31. Xian W, McKeon F, Vincent M, Crum C and Ho KY: Methods and reagents for detection and treatment of esophageal metaplasia. Patent WO2012044992A2. Filed September 30, 2011; issued April 5, 2012.

32. Iyer K, Evans C, Clemons T, Fitzgerald M, Dunlop S, Luzinov I and Zdyrko B: Multifunctional nanoparticles. Patent WO2012075533A1. Filed December 7, 2011; issued June 14, 2012.

33. Zhao Y: Nanoparticles and nanoparticle compositions. Patent WO2011130114A1. Filed April 8, 2011; issued October 20, 2011.

34. Bayford RH, Roitt IM, Rademacher TW, Demosthenous A and Iles RK: Detection of cancer. Patent WO2010052503A1. Filed November 6, 2009; issued May 14, 2010.

35. Zhu X, Lu N, Zhou Y, Xuan S, Zhang J, Giampieri F, Zhang Y, Yang F, Yu R, Battino M, et al: Targeting pancreatic cancer cells with peptide-functionalized polymeric magnetic nanoparticles. Int J Mol Sci 20: 2988, 2019.

36. Wei QY, He KM, Chen JL, Xu YM and Lau ATY: Phytofabrication of nanoparticles as novel drugs for anticancer applications. Molecules 24: 4246, 2019.
37. Fang L, Fan $\mathrm{H}$, Guo C, Cui L, Zhang P, Mu H, Xu H, Zhao F and Chen D: Novel mitochondrial targeting multifunctional surface charge-reversal polymeric nanoparticles for cancer treatment. J Biomed Nanotechnol 15: 2151-2163, 2019.

38. Cavalcante GC, Schaan AP, Cabral GF, Santana-da-Silva MN, Pinto P, Vidal AF and Ribeiro-Dos-Santos Â: A cell's fate: An Overview of the molecular biology and genetics of apoptosis. Int J Mol Sci 20: 4133, 2019.

39. Xia M, Zhang Y, Jin K, Lu Z, Zeng Z and Xiong W: Communication between mitochondria and other organelles: A brand-new perspective on mitochondria in cancer. Cell Biosci 9: 27, 2019.

40. Cho YL, Tan HWS, Saquib Q, Ren Y, Ahmad J, Wahab R, He W, Bay BH and Shen HM: Dual role of oxidative stress-JNK activation in autophagy and apoptosis induced by nickel oxide nanoparticles in human cancer cells. Free Radic Biol Med 153: 173-186, 2020.

41. Chattopadhyay S, Dash SK, Tripathy S, Das B, Kar Mahapatra S, Pramanik P and Roy S: Cobalt oxide nanoparticles induced oxidative stress linked to activation of TNF- $\alpha /$ caspase-8/p38-MAPK signaling in human leukemia cells. J Appl Toxicol 35: 603-613, 2015.

42. Dai DF, Chiao YA, Martin GM, Marcinek DJ, Basisty N, Quarles EK and Rabinovitch PS: Mitochondrial-targeted catalase: Extended longevity and the roles in various disease models. Prog Mol Biol Transl Sci 146: 203-241, 2017.

43. Li Y and Ju D: The role of autophagy in nanoparticles-induced toxicity and its related cellular and molecular mechanisms. Adv Exp Med Biol 1048: 71-84, 2018

44. Cordani M and Somoza Á: Targeting autophagy using metallic nanoparticles: A promising strategy for cancer treatment. Cell Mol Life Sci 76: 1215-1242, 2019.

45. Wallace DC: Mitochondria and cancer. Nat Rev Cancer 12: 685-698, 2012

46. GuoR,PengH,Tian Y,Shen S and Yang W: Mitochondria-targeting magnetic composite nanoparticles for enhanced phototherapy of cancer. Small 12: 4541-4552, 2016

47. Yin B, Li KHK, Ho LWC, Chan CKW and Choi CHJ: Toward understanding in vivo sequestration of nanoparticles at the molecular level. ACS Nano 12: 2088-2093, 2018.

48. Bader JE, Enos RT, Velázquez KT, Carson MS, Nagarkatti M, Nagarkatti PS, Chatzistamou I, Davis JM, Carson JA, Robinson CM, et al: Macrophage depletion using clodronate liposomes decreases tumorigenesis and alters gut microbiota in the AOM/DSS mouse model of colon cancer. Am J Physiol Gastrointest Liver Physiol 314: G22-G31, 2018.

49. Kim YJ, Perumalsamy H, Castro-Aceituno V, Kim D, Markus J, Lee S, Kim S, Liu Y and Yang DC: Photoluminescent and self-assembled hyaluronic acid-zinc oxide-ginsenoside Rh2 nanoparticles and their potential Caspase-9 apoptotic mechanism towards cancer cell lines. Int J Nanomedicine 14: 8195-8208, 2019.

50. Wang H, Zhang F, Wen H, Shi W, Huang Q, Huang Y, Xie J, Li P, Chen J, Qin L, et al: Tumor- and mitochondria-targeted nanoparticles eradicate drug resistant lung cancer through mitochondrial pathway of apoptosis. J Nanobiotechnology 18: 8, 2020.

51. Gong N, Ma X, Ye X, Zhou Q, Chen X, Tan X, Yao S, Huo S, Zhang T, Chen S, et al: Carbon-dot-supported atomically dispersed gold as a mitochondrial oxidative stress amplifier for cancer treatment. Nat Nanotechnol 14: 379-387, 2019.

52. Sun H, Jiang C, Wu L, Bai X and Zhai S: Cytotoxicity-related bioeffects induced by nanoparticles: the role of surface chemistry. Front Bioeng Biotechnol 7: 414, 2019.

53. Kurtz-Chalot A, Villiers C, Pourchez J, Boudard D, Martini M, Marche PN, Cottier M and Forest V: Impact of silica nanoparticle surface chemistry on protein corona formation and consequential interactions with biological cells. Mater Sci Eng C 75: 16-24, 2017.

54. Wang Z, Wang C, Liu S, He W, Wang L, Gan J, Huang Z, Wang Z, Wei $\mathrm{H}$, Zhang J, et al: Specifically formed corona on silica nanoparticles enhances transforming growth factor $\beta 1$ activity in triggering lung fibrosis. ACS Nano 11: 1659-1672, 2017.

55. Vasileiou PVS, Evangelou K, Vlasis K, Fildisis G, Panayiotidis MI, Chronopoulos E, Passias PG, Kouloukoussa M, Gorgoulis VG and Havaki S: Mitochondrial homeostasis and cellular senescence. Cells 8: 686, 2019.

56. Burgos-Morón E, Abad-Jiménez Z, Marañón AM, Iannantuoni F, Escribano-López I, López-Domènech S, Salom C, Jover A, Mora V, Roldan I, et al: Relationship between oxidative stress, ER stress, and inflammation in type 2 diabetes: The Battle Continues. J Clin Med 8: 1385, 2019. 
57. Balan C, Chis MI, Rachisan AL and Baia M: A vibrational study of inulin by means of experimental and theoretical methods. J Mol Struct 1164: 84-88, 2018.

58. Picos A, Rachisan AL and Dadarlat A: Minimally invasive denta treatment using composites and ceramics in GERD diagnoses patients. Mater Plast 55: 252-254, 2018.

59. Lin LS, Wang JF, Song J, Liu Y, Zhu G, Dai Y, Shen Z, Tian R, Song J, Wang Z, et al: Cooperation of endogenous and exogenous reactive oxygen species induced by zinc peroxide nanoparticles to enhance oxidative stress-based cancer therapy. Theranostics 9: 7200-7209, 2019.
60. Sur S, Nakanishi H, Flaveny C, Ippolito JE, McHowat J, Ford DA and Ray RB: Inhibition of the key metabolic pathways, glycolysis and lipogenesis, of oral cancer by bitter melon extract. Cell Commun Signal 17: 131, 2019.

61. Huo D, Zhu J, Chen G, Chen Q, Zhang C, Luo X, Jiang W, Jiang X, $\mathrm{Gu} \mathrm{Z}$ and $\mathrm{Hu} \mathrm{Y}$ : Eradication of unresectable liver metastasis through induction of tumour specific energy depletion. Nat Commun 10: 3051, 2019. 